\title{
The physical conditions and oxygen and nitrogen abundance of 36 SBS galaxies from the SDSS DR7
}

\author{
M. V. Gyulzadyan ${ }^{1}$ and V. Z. Adibekyan ${ }^{2}$ \\ ${ }^{1}$ Byurakan Astrophysical Observatory, Armenia \\ email: mgyulz@bao.sci.am \\ ${ }^{2}$ Centro de Astrofisica da Universidade do Porto, Portugal
}

\begin{abstract}
Physical conditions and oxygen and nitrogen abundances in 36 SBS UV-excess and/or emission-line galaxies from the SDSS DR7 were determined. We have found that SBS $0808+578$ is AGN. The others are HII galaxies or HII regions in galaxies. For all objects the oxygen abundance $12+\log (\mathrm{O} / \mathrm{H})$ lies in the range of $7.85 \div 8.61$ and $\log (\mathrm{N} / \mathrm{O})$ ratio in the range of $-1.45 \div-0.4$. They occupy the same area in the diagram $\mathrm{N} / \mathrm{O} \mathrm{O} / \mathrm{H}$ as the high-excitation HII regions. We found no extremely metal-deficient galaxy. Using $\mathrm{H}$-alpha fluxes star formation rates (SFR) for our samples galaxies were determined. Determined SFRs, being in the range of $0.001 \div 6$ o year $^{-} 1$, are similar of that observed in typical star forming regions in spiral and irregular galaxies.
\end{abstract}

Keywords. surveys - galaxies: active — galaxies: Seyfert — galaxies: starburst

\section{Introduction}

Accurate metallicities play a key role in many investigations of galaxies. Gas-phase oxygen and nitrogen abundances are broadly used to measure these metallicities. It is believed (e. g., Stasinska 2006) that emission lines due to photoionization by massive stars are the most powerful indicator of the chemical composition of galaxies, both in the low- and intermediate-redshift universe.

The oxygen abundance can be considered as a tool to investigate the evolution of galaxies. For example, the value of oxygen abundance in a galaxy combined with the value of the gas mass fraction can tell us about the efficiency of mass exchange between a galaxy and its environment (Pilyugin \& Ferrini, 2000)

\section{The samples}

The Seventh Data Release of the Sloan Digital Sky Survey (SDSS), marking the completion of the original goals of the SDSS and the end of the phase known as SDSS-II. It includes $11663 \mathrm{deg}^{2}$ of imaging data, with most of the $\sim 2000 \mathrm{deg}^{2}$ increment over the previous data release lying in regions of low Galactic latitude. The catalog contains fiveband photometry for 357 million distinct objects. The survey also includes a condition of the best of these data, going roughly two magnitudes fainter than the main survey over $250 \mathrm{deg}^{2}$. The survey has completed spectroscopy over $9380 \mathrm{deg}^{2}$; the spectroscopy is now complete over a large contiguous area of the Northern Galactic Cap, closing the gap that was present in previous data releases. There are over 1.6 million spectra in total, including 930,000 galaxies, 120,000 quasars, and 460,000 stars. 
Using spectroscopic information from the Data Release 7 of Sloan Digital Sky Survey (SDSS DR7), we analyzed the SBS galaxies and found 66 new observed spectra for SBS galaxies. The equivalent widths of $\mathrm{H}_{\beta}\left(\mathrm{EW}\left(\mathrm{H}_{\beta}\right)\right)$ lines are more then 2 for only $36 \mathrm{SBS}$ galaxies, which were used for detailed investigation.

The Second Byurakan Survey (SBS) is well-known combined survey, which uses the technique of selecting objects according to their excess UV emission as well the presence of emission lines in their low-dispersion, objective prism spectra (Stepanian 2005).

In the $[\mathrm{NII}] / \mathrm{H}_{\beta}-[\mathrm{OIII}] / \mathrm{H}_{\alpha}$ classification diagram, the SBS $0808+578$ galaxy lay in the AGN region, wile the remaining 35 galaxies lay in the $\mathrm{SF}$ region.

\section{The physical conditions and element abundance}

The physical conditions and element abundance are derived from the redding correcting emission line fluxes using the electron temperature. We use indirect method, because the lines of [OIII] $\lambda 4363$ are not detectable or too weak to be detected in our sample.

Having the fluxes of the $\mathrm{H}_{\alpha}$ line as corrected for stellar absorption and dust obscuration, we determined the SFR.

In HII regions the emission lines of hydrogen are an indicator of a young star population, since they are formed after absorbing and reprocessing the radiation from these stars beyond the Lyman series limit. These lines were used to calculate the number of ionizing stars.

\section{Conclusions}

Studies the spectra of the SBS 36 galaxies from SDSS DR7 show that SBS $0808+578$ is AGN. The others are the star forming HII galaxies or HII regions in galaxies.

The redshifts of these galaxies lies between $0.004 \div 1.01795$. Their blue and near infrared absolute magnitudes lies between $-24.8^{m} \div-23.9^{m}\left(<\mathrm{M}(\mathrm{B})>=-19.7^{m} \pm 1.9^{m}\right)$; and $-25^{m} \div-18^{m}\left(<\mathrm{M}(\mathrm{K})>=-22.8^{m} \pm 1.3^{m}\right)$.

The star formation rate for investigated galaxies is between $0.001 \div 6 \mathrm{M} \mathrm{year}^{-1}$. The surface density of the star formation rate in the nuclear regions lies between $0.02 \div 1.65$. For 5 regions it is more then 1 . The number of $\mathrm{O} 7 \mathrm{~V}$ stars $\mathrm{N}(\mathrm{O} 7 \mathrm{~V})$ for 11 galaxies is more then 10,000. The highest star formation activity is observed in the galaxy SBS $0830+590$. The value of SFR and $\mathrm{N}(\mathrm{O} 7 \mathrm{~V})$ is $6 \mathrm{M}_{\text {year }}{ }^{-1}$ and 54478 respectively. For HII galaxies we determinate the electron temperatures, oxygen and nitrogen abundance too.

Of the HII regions studied here, none had a very low metallicity $(12+\log (\mathrm{O} / \mathrm{H})<7.6$. i.e., $\mathrm{Z}<\mathrm{Z} / 12)$. The oxygen abundance in this regions lies $7.85 \div 8.6 \mathrm{Log}(\mathrm{N} / \mathrm{O})$ for these nucleus lies $-1.45 \div-0.4$. For a given metallicity they have the same values of $\log (\mathrm{N} / \mathrm{O})$ as high-excitation HII regions (Izotov et al., 2004; Izotov et al., 2006). Most likely, the age of these HII regions exceeds the 100-300 million years, required for the enrichment in nitrogen by intermediate-mass stars.

Data from the seventh database of the Sloan Digital Sky Survey (SDSS DR7), which is available for open access at http://www.sdss.org/dr7, have been used in this paper.

\section{References}

Izotov, Y. I., Stasiska, G., Guseva, N. G., \& Tuan, T. X. 2004. A\&A 415, 87

Izotov, Y. I., Stasiska, G., Meynet, G., Guseva, N. G., \& Tuan, T. X. 2006, A\&A 448, 955

Pilyugin, L. S. \& Ferrini, F. 2000, A\&A 354,874

Stasiska, G. 2006, A\&A 454, L127

Stepanyan, J. 2005, Rev. Mexicana AyA 41, 155 